\title{
THERMAL COHERENT STATES, A BROADER CLASS OF MIXED COHERENT STATES, AND GENERALIZED THERMO-FIELD DYNAMICS
}

\author{
RAYMOND F. BISHOP \\ School of Physics and Astronomy, The University of Manchester, \\ Sackville Street Building, P.O. Box 88, Manchester, M60 1QD, UK \\ (email: raymond.bishop@manchester.ac.uk) \\ APOSTOLOS VOURDAS \\ Department of Computing, University of Bradford, \\ Richmond Road, Bradford, West Yorkshire, BD7 1DP, UK
}

\begin{abstract}
We introduce coherent mixed states (or thermal coherent states) associated with the displaced harmonic oscillator at nonzero temperature $(T \neq 0)$, as a "random" (or "thermal" or "noisy") basis in Hilbert space. A resolution of the identity for these states is proven and is used to generalize the usual pure $(T=0)$ coherent state formalism to the mixed $(T \neq 0)$ case. This new formalism for thermal coherent states is then further generalized to a broader class of so-called negative-binomial mixed states. It is known that the negative-binomial distribution is itself intimately related to the discrete series of $\mathrm{SU}(1,1)$ representations. We consider the pure $\mathrm{SU}(1,1)$ coherent states in the two-mode harmonic oscillator space, and show how our negative-binomial mixed states arise from taking the partial trace with respect to one of these two modes. This observation is then used to show how the formalism of thermo-field dynamics may be generalized to a correspondingly much broader negative-binomial-field dynamics, which we expect to have many uses.
\end{abstract}

Keywords: Glauber coherent states; $\mathrm{SU}(1,1)$ coherent states; mixed coherent states; thermal coherent states; negative-binomial mixed coherent states; generalized therm-field dynamics; negative-binomial-field dynamics; coherence and decoherence.

\section{Introduction}

The pure Glauber (harmonic oscillator) coherent states provide a very useful basis for many purposes. They are complete since an arbitrary state in the Hilbert space may be expanded in terms of them. Some of their main properties are discussed in Sec. 2. Our main aim in the present paper is to generalize the Glauber coherent states in several directions. The first involves an extension to the idea of mixed coherent states, and we show in particular in Sec. 3 that we may introduce a set of thermal mixed coherent states that completely generalize the usual pure Glauber formalism to the mixed case where the oscillator is in contact with a heat bath at a nonzero temperature $(T=0)$. We show, for example how the thermal 
coherent states permit a generalization to the $T \neq 0$ case of the usual $P$ - and $Q$-representations of operators.

A second generalization in Sec. 4 is to consider various (one- and two-mode) realizations of the pure (squeezed) coherent states associated with the group SU $(\mathbf{1}, 1)$. One of these is associated with the so-called $k$-squeezed states of a single boson (or mode), and this introduces us to the pure SU $(1,1)$ negative-binomial coherent states. These are then further generalized in Sec. 5 to the so-called negative-binomial mixed coherent states, which are associated with the discrete series of representations of $\mathrm{SU}(1,1)$ characterized by the quantum number $k=\frac{1}{2}, 1, \frac{3}{2}, \ldots$. We show that the thermal coherent states are the particular limiting case $k=\frac{1}{2}$ of this much larger class.

In Sec. 4 we also consider squeezed states associated with two different modes (or bosons). The Hilbert space of such a two-mode oscillator can be expressed as a direct sum of an infinite number of subspaces, each of which is a particular $k$-representation of $\mathrm{SU}(1,1)$. In Sec. 6 we reconsider the pure $\mathrm{SU}(1,1)$ coherent states in the twoboson Hilbert space, and show that the partial trace over one of the two boson Hilbert spaces leads, rather miraculously, to our previous negative-binomial mixed states of Sec. 5. We hence show how thermo-field dynamics may be generalized to a correspondingly much broader "negative-binomial-field dynamics," which we expect to have many uses. The work is summarized in Sec. 7 , where we also briefly consider potential applications.

\section{Single-Boson Pure (Glauber) Coherent States}

Consider a single-mode harmonic oscillator with Hamiltonian,

$$
\hat{H}_{0}=\frac{\hat{p}^{2}}{2 m}+\frac{1}{2} m \omega^{2} \hat{x}^{2}=\hbar \omega\left(\hat{a}_{0}^{\dagger} \hat{a}_{0}+\frac{1}{2}\right) \equiv \hbar \omega\left(\hat{n}_{0}+\frac{1}{2}\right),
$$

in terms of the usual boson creation and destruction operators, $\hat{a}_{0}^{\dagger}$ and $\hat{a}_{0}$ respectively,

$$
\hat{a}_{0} \equiv \sqrt{\frac{m \omega}{2 \hbar}}\left(\hat{x}+\frac{\mathrm{i}}{m \omega} \hat{p}\right)
$$

and which hence obey the usual canonical commutation relations, $\left[\hat{a}_{0}, \hat{a}_{0}^{\dagger}\right]=\hat{\mathbb{1}}$. The set of orthonormal number eigenstates,

$$
|n\rangle \equiv(n !)^{-1 / 2}\left(\hat{a}_{0}^{\dagger}\right)^{n}|0\rangle ; n=0,1,2, \ldots,
$$

is defined in terms of the normalized vacuum state $|0\rangle, \hat{a}_{0}|0\rangle=0$. They obey the completeness relation,

$$
\sum_{n=0}^{\infty}|n\rangle\langle n|=\hat{\mathbb{1}}
$$


The normalized state $|z\rangle \equiv|x, p\rangle$ is a Glauber coherent state if it is a right eigenstate of $\hat{a}_{0}$,

$$
\hat{a}_{0}|z\rangle=z|z\rangle \Longleftrightarrow\left(\hat{x}+\frac{\mathrm{i}}{m \omega} \hat{p}\right)|x, p\rangle=\left(x+\frac{\mathrm{i}}{m \omega} p\right)|x, p\rangle ; x, p \in \mathbb{R}
$$

with

$$
z \equiv \sqrt{\frac{m \omega}{2 \hbar}}\left(x+\frac{\mathrm{i}}{m \omega} p\right) ; x, p \in \mathbb{R} .
$$

Expanding $|z\rangle$ in terms of number eigenstates, we easily find

$$
|z\rangle=\exp \left(-\frac{1}{2}|z|^{2}\right) \sum_{n=0}^{\infty} \frac{z^{n}}{\sqrt{n !}}|n\rangle=\exp \left(-\frac{1}{2}|z|^{2}\right) \exp \left(z \hat{a}_{0}^{\dagger}\right)|0\rangle .
$$

Alternatively, by making use of the disentangling relation for the Heisenberg-Weyl group,

$$
\exp \left(z \hat{a}_{0}^{\dagger}-z^{*} \hat{a}_{0}\right)=\exp \left(-\frac{1}{2}|z|^{2}\right) \exp \left(z \hat{a}_{0}^{\dagger}\right) \exp \left(-z^{*} \hat{a}_{0}\right)
$$

we may write $|z\rangle$ in terms of the unitary Weyl displacement operator,

$$
\hat{D}(z) \equiv \exp \left(z \hat{a}_{0}^{\dagger}-z^{*} \hat{a}_{0}\right) \Longleftrightarrow \hat{D}(x, p) \equiv \exp [\mathbf{i}(p \hat{x}-x \hat{p}) / \hbar]
$$

as

$$
|z\rangle=\hat{D}(z)|0\rangle \Longleftrightarrow|x, p\rangle=\hat{D}(x, p)|0\rangle
$$

We may readily check that the mode of action of the Weyl displacement operator on the elementary group operators is as follows,

$$
\begin{array}{cl}
\hat{D}(z) \hat{a}_{0} \hat{D}^{\dagger}(z)=\hat{a}_{0}-z \hat{\mathbb{1}} \equiv \hat{a}_{z} ; & \hat{D}(z) \hat{a}_{0}^{\dagger} \hat{D}^{\dagger}(z)=\hat{a}_{0}^{\dagger}-z^{*} \hat{\mathbb{1}} \equiv \hat{a}_{z}^{\dagger}, \\
\hat{D}\left(x_{0}, p_{0}\right) \hat{x} \hat{D}^{\dagger}\left(x_{0}, p_{0}\right)=\hat{x}-x_{0} \hat{\mathbb{1}} ; & \hat{D}\left(x_{0}, p_{0}\right) \hat{p} \hat{D}^{\dagger}\left(x_{0}, p_{0}\right)=\hat{p}-p_{0} \hat{\mathbb{1}} .
\end{array}
$$

Very importantly, the Glauber coherent states provide a resolution of the identity operator,

$$
\iint_{C} \frac{\mathrm{d}^{2} z}{\pi}|z\rangle\left\langle z\left|=\hat{\mathbb{1}} \Longleftrightarrow \int_{-\infty}^{\infty} \int_{-\infty}^{\infty} \frac{\mathrm{d} x \mathrm{~d} p}{2 \pi \hbar}\right| x, p\right\rangle\langle x, p|=\hat{\mathbb{1}},
$$

despite being massively overcomplete, where the integration over $z$ is over the entire complex plane, $C$, and where the integration element is $\mathrm{d}^{2} z \equiv \mathrm{d} z_{\mathrm{R}} \mathrm{d} z_{\mathrm{I}}=\mathrm{d} z \mathrm{~d} z^{*} / 2 \mathrm{i}$.

More general coherent states $|\ell\rangle$ are required to satisfy the two conditions that they are strongly continuous functions of the label $\ell \in L$, where the (often multidimensional) label space $L$ is continuous, and that they provide a resolution of the identity operator,

$$
\int_{L} \delta \ell|\ell\rangle\langle\ell|=\hat{\mathbb{1}}
$$


with a suitable choice of positive measure $\delta \ell$. We note that $\mathrm{Eq},(14)$ implies that all admissible wave functions $\langle\ell \mid \Psi\rangle$ satisfy the nontrivial homogeneous integral equation,

$$
\langle\ell \mid \Psi\rangle=\int_{L} \delta \ell^{\prime}\left\langle\ell \mid \ell^{\prime}\right\rangle\left\langle\ell^{\prime} \mid \Psi\right\rangle
$$

involving the reproducing kernel $K\left(\ell, \ell^{\prime}\right) \equiv\left\langle\ell \mid \ell^{\prime}\right\rangle=\left\langle\ell^{\prime} \mid \ell\right\rangle^{*}$. For the Glauber coherent states we may use Eq. (7) to show that

$$
K\left(z, z^{\prime}\right) \equiv\left\langle z \mid z^{\prime}\right\rangle=\exp \left(-\frac{1}{2}|z|^{2}-\frac{1}{2}\left|z^{\prime}\right|^{2}+z^{*} z^{\prime}\right)
$$

\subsection{Glauber coherent states and phase-space distribution functions}

It is well known in quantum optics that all operators belonging to the trace class (i.e., all operators $\hat{\Theta}$ for which $\operatorname{Tr} \hat{\Theta}<\infty$ ) are uniquely specified by either their $Q$ or their (Glauber-Sudarshan) $P$-representation,

$$
\begin{aligned}
Q(\hat{\Theta} ; z) \equiv\langle z|\hat{\Theta}| z\rangle & \Longleftrightarrow Q(\hat{\Theta} ; x, p) \equiv\langle x, p|\hat{\Theta}| x, p\rangle \\
\hat{\Theta} \equiv \iint_{C} \frac{\mathrm{d}^{2} z}{\pi} P(\hat{\Theta} ; z)|z\rangle\langle z| & \Longleftrightarrow \hat{\Theta} \equiv \int_{-\infty}^{\infty} \int_{-\infty}^{\infty} \frac{\mathrm{d} x \mathrm{~d} p}{2 \pi \hbar} P(\hat{\Theta} ; x, p)|x, p\rangle\langle x, p| .
\end{aligned}
$$

Equations (16)-(18) readily yield a relationship between these two representations,

$$
Q(\hat{\Theta} ; z)=\iint_{C} \frac{\mathrm{d}^{2} z^{\prime}}{\pi} P\left(\hat{\Theta} ; z^{\prime}\right) \mathrm{e}^{-\left(z-z^{\prime}\right)^{2}}=\exp \left(\frac{1}{4} \Delta_{z}\right) P(\hat{\Theta} ; z),
$$

in terms of the two-dimensional Laplacian operator,

$$
\Delta_{z} \equiv 4 \frac{\partial^{2}}{\partial z \partial z^{*}}=\frac{\partial^{2}}{\partial z_{\mathrm{R}}^{2}}+\frac{\partial^{2}}{\partial z_{\mathrm{I}}^{2}}=2 \hbar\left(\frac{1}{m \omega} \frac{\partial^{2}}{\partial x^{2}}+m \omega \frac{\partial^{2}}{\partial p^{2}}\right) .
$$

For an arbitrary function $f(z)=f\left(z, z^{*}\right)$ we now introduce its two-dimensional Fourier transform,

$$
\tilde{f}(w) \equiv \iint_{C} \frac{\mathrm{d}^{2} z}{\pi} \mathrm{e}^{\mathrm{i} z \cdot w} f(z) \Longleftrightarrow f(z)=\iint_{C} \frac{\mathrm{d}^{2} w}{4 \pi} \mathrm{e}^{-\mathrm{i} z \cdot w} \tilde{f}(w),
$$

where $z \cdot w \equiv \frac{1}{2}\left(z w^{*}+z^{*} w\right)=z_{\mathrm{R}} w_{\mathrm{R}}+z_{\mathrm{I}} w_{\mathrm{I}}$. Alternatively, if we express $w$ as

$$
w \equiv \sqrt{\frac{2}{m \hbar \omega}}(-P+\mathrm{i} m \omega X) ; X, P \in \mathbb{R},
$$

as the analogue of Eq. (6), then Eq. (21) may be expressed in the equivalent forms,

$$
\begin{aligned}
\tilde{f}(X, P) & \equiv \int_{-\infty}^{\infty} \int_{-\infty}^{\infty} \frac{\mathrm{d} x \mathrm{~d} p}{2 \pi \hbar} \mathrm{e}^{\mathrm{i}(X p-P x) / \hbar} f(x, p) \Longleftrightarrow \\
f(x, p) & =\int_{-\infty}^{\infty} \int_{-\infty}^{\infty} \frac{\mathrm{d} X \mathrm{~d} P}{2 \pi \hbar} \mathrm{e}^{\mathrm{i}(x P-p X) / \hbar} \tilde{f}(X, P) .
\end{aligned}
$$


An equivalent form of Eq. (19) may thus be given as

$$
\tilde{Q}(\hat{\Theta} ; w)=\exp \left(-\frac{1}{4}|w|^{2}\right) \tilde{P}(\hat{\Theta} ; w) .
$$

Finally, we define the Weyl representation of $\hat{\Theta}$ as follows,

$$
\tilde{W}(\hat{\Theta} ; w) \equiv \operatorname{Tr}\left\{\hat{\Theta} \hat{D}\left(\frac{1}{2} \mathbf{i} w\right)\right\} \text {. }
$$

Its Fourier transform, $W(\hat{\Theta} ; z)$, is the Wigner or $W$-representation of $\hat{\Theta}$. In terms of position eigenstates $|x\rangle_{x}$, where $\hat{x}|x\rangle_{x}=x|x\rangle_{x}$, we may readily derive the more familiar forms,

$$
\begin{aligned}
& W(\hat{\Theta} ; z)=\int_{-\infty}^{\infty} \mathrm{d} X{ }_{x}\left\langle\sqrt{2} z_{\mathrm{R}}+\frac{1}{2} X|\hat{\Theta}| \sqrt{2} z_{\mathrm{R}}-\frac{1}{2} X\right\rangle_{x} \exp \left(-\mathrm{i} \sqrt{2} z_{\mathrm{I}} X\right), \\
& \tilde{W}(\hat{\Theta} ; w)=\int_{-\infty}^{\infty} \mathrm{d} x_{x}\left\langle x+2^{-3 / 2} w_{\mathrm{I}}|\hat{\Theta}| x-2^{-3 / 2} w_{\mathrm{I}}\right\rangle_{x} \exp \left(\mathrm{i} w_{\mathrm{R}} x / \sqrt{2}\right),
\end{aligned}
$$

in units where we have now put $m=\omega=\hbar=1$, or equivalently,

$$
\begin{gathered}
W(\hat{\Theta} ; x, p)=\int_{-\infty}^{\infty} \mathrm{d} X{ }_{x}\left\langle x+\frac{1}{2} X|\hat{\Theta}| x-\frac{1}{2} X\right\rangle_{x} \exp (-\mathrm{i} p X / \hbar), \\
\tilde{W}(\hat{\Theta} ; X, P)=\int_{-\infty}^{\infty} \mathrm{d} x_{x}\left\langle x+\frac{1}{2} X|\hat{\Theta}| x-\frac{1}{2} X\right\rangle_{x} \exp (-\mathrm{i} P x / \hbar) .
\end{gathered}
$$

We also easily find the following relations between the $P_{-}, Q-$, and $W-$ representations,

$$
\begin{aligned}
& Q(\hat{\Theta} ; z)=\exp \left(+\frac{1}{8} \Delta_{z}\right) W(\hat{\Theta} ; z) \Longleftrightarrow \tilde{Q}(\hat{\Theta} ; w)=\exp \left(-\frac{1}{8}|w|^{2}\right) \tilde{W}(\hat{\Theta} ; w), \\
& P(\hat{\Theta} ; z)=\exp \left(-\frac{1}{8} \Delta_{z}\right) W(\hat{\Theta} ; z) \Longleftrightarrow \tilde{P}(\hat{\Theta} ; w)=\exp \left(+\frac{1}{8}|w|^{2}\right) \tilde{W}(\hat{\Theta} ; w) .
\end{aligned}
$$

Eq. (28) also implies our previous Eq. (19), which may be rewritten in the form,

$$
Q(\hat{\Theta} ; z)=\exp \left(+\frac{1}{4} \Delta_{z}\right) P(\hat{\Theta} ; z) \Longleftrightarrow \tilde{Q}(\hat{\Theta} ; w)=\exp \left(-\frac{1}{4}|w|^{2}\right) \tilde{P}(\hat{\Theta} ; w)
$$

\section{Mixed Thermal Coherent States}

Our basic idea ${ }^{1}$ to extend the pure-state formalism outlined in Sec. 2 above is now to use a set of mixed states as a basis in Hilbert space. Previously we used the pure coherent states $|z\rangle$ with projectors $\hat{\Pi}(z) \equiv|z\rangle\langle z|, \hat{\Pi}^{2}(z)=\hat{\Pi}(z)$. The resolution of the identity operator given by Eq. (13) then allowed us to resolve an arbitrary state $|f\rangle$ as

$$
|f\rangle=\iint_{C} \frac{\mathrm{d}^{2} z}{\pi}\langle z \mid f\rangle|z\rangle
$$

A mixed state is described by a density operator $\hat{\rho}$ with eigenstates $\left|e^{(n)}\right\rangle$ and corresponding eigenvalues $p_{n}$,

$$
\hat{\rho}=\sum_{n=0}^{\infty} p_{n}\left|e^{(n)}\right\rangle\left\langle e^{(n)}\right| ; \sum_{n=0}^{\infty} p_{n}=1 ; 0 \leq p_{i} \leq 1, \forall i
$$


It clearly represents a set of states $\left\{\left|e^{(n)}\right\rangle\right\}$ with a probability distribution $\left\{p_{n}\right\}$. Thus, the idea of using mixed states as a basis replaces the "fixed" vectors usually used with "noisy" or "random" vectors.

In order to make these ideas concrete we first consider the thermodynamic density operator for a canonical ensemble of single-frequency $(\omega)$ harmonic oscillators, with Hamiltonian $\hat{H}_{0}$ given by Eq. (1), in thermal equilibrium at a temperature $T=\left(k_{\mathrm{B}} \beta\right)^{-1}$,

$$
\hat{\rho}_{0}(\beta) \equiv \exp \left(-\beta \hat{H}_{0}\right) / \operatorname{Tr}\left\{\exp \left(-\beta \hat{H}_{0}\right)\right\}=\left(1-\mathrm{e}^{-\beta \hbar \omega}\right) \exp \left(-\beta \hbar \omega \hat{a}_{0}^{\dagger} \hat{a}_{0}\right) .
$$

Of particular importance to us, as we shall see below, is the displaced linear harmonic oscillator with Hamiltonian given by

$$
\hat{H}_{z} \equiv \hat{D}(z) \hat{H}_{0} \hat{D}^{\dagger}(z)=\hbar \omega\left[\left(\hat{a}_{0}^{\dagger}-z^{*} \hat{\mathbb{1}}\right)\left(\hat{a}_{0}-z \hat{\mathbb{1}}\right)+\frac{1}{2}\right] \equiv \hbar \omega\left(\hat{a}_{z}^{\dagger} \hat{a}_{z}+\frac{1}{2}\right),
$$

with the various operators as defined previously in Sec. 1. Its density operator, which represents the Boltzmann distribution for the monochromatic oscillator, is given by

$$
\hat{\rho}(z ; \beta) \equiv \hat{D}(z) \hat{\rho}_{0}(\beta) \hat{D}^{\dagger}(z)=\left(1-\mathrm{e}^{-\beta \hbar \omega}\right) \exp \left(-\beta \hbar \omega \hat{a}_{z}^{\dagger} \hat{a}_{z}\right)
$$

and hence where $\hat{\rho}(0 ; \beta) \equiv \hat{\rho}_{0}(\beta)$, or equivalently, as

$$
\hat{\rho}(z ; \beta)=\sum_{n=0}^{\infty} p_{n}(\beta)|n ; z\rangle\langle n ; z| ;
$$

where

$$
|n ; z\rangle \equiv \hat{D}(z)|n\rangle ; p_{n}(\beta) \equiv\left(1-\mathrm{e}^{-\beta \hbar \omega}\right) \mathrm{e}^{-n \beta \hbar \omega} .
$$

Its zero-temperature limit just represents the above coherent-state projector $\hat{\Pi}(z)$,

$$
\hat{\rho}(z ; \beta) \underset{\beta \rightarrow \infty}{\longrightarrow}|z\rangle\langle z| \equiv \hat{\Pi}(z) .
$$

Using the definitions of Eqs. (17) and (18) we may easily calculate its $Q$ - and $P$ representations,

$$
\begin{aligned}
& Q(\hat{\rho}(\zeta ; \beta) ; z)=\left(1-\mathrm{e}^{-\beta \hbar \omega}\right) \exp \left[-\left(1-\mathrm{e}^{-\beta \hbar \omega}\right)|z-\zeta|^{2}\right], \\
& P(\hat{\rho}(\zeta ; \beta) ; z)=\left(\mathrm{e}^{\beta \hbar \omega}-1\right) \exp \left[-\left(\mathrm{e}^{\beta \hbar \omega}-1\right)|z-\zeta|^{2}\right] .
\end{aligned}
$$

Using Eqs. (38) and (39) we can trivially prove the relations

$$
\iint_{C} \frac{\mathrm{d}^{2} \zeta}{\pi} Q(\hat{\rho}(\zeta ; \beta) ; z)=1=Q(\hat{\mathbb{1}} ; z) ; \iint_{C} \frac{\mathrm{d}^{2} \zeta}{\pi} P(\hat{\rho}(\zeta ; \beta) ; z)=1=P(\hat{\mathbb{1}} ; z) ;
$$

From Eq. (40) follows immediately the extremely important resolution of the identity,

$$
\iint_{C} \frac{\mathrm{d}^{2} z}{\pi} \hat{\rho}(z ; \beta)=\hat{\mathbb{1}}
$$


which is the generalization to nonzero temperatures of Eq. (13). Eq. (41) allows us to resolve an arbitrary state $|f\rangle$ as

$$
|f\rangle=\sum_{n=0}^{\infty} p_{n} \iint_{C} \frac{\mathrm{d}^{2} z}{\pi}\langle n ; z \mid f\rangle|n ; z\rangle,
$$

which is a generalization of the pure coherent-state expansion of Eq. (30) to the "noisy" thermal basis.

\subsection{Relationship to the Glauber-Lachs formalism in quantum optics}

Before proceeding further it is worthwhile to pause for a moment to point out the connection between our density operator $\hat{\rho}(z ; \beta)$ and the Glauber-Lachs formalism in quantum optics for mixtures of incoherent (or, loosely, thermal) and coherent radiation. Thus, it has been shown by Glauber ${ }^{2}$ that the $P$-representation for a field which is a superposition of two separate fields with individual $P$-representations $P_{1}(z)$ and $P_{2}(z)$ is given by their convolution,

$$
P(z)=\iint_{C} \frac{\mathrm{d}^{2} z^{\prime}}{\pi} P_{1}\left(z^{\prime}\right) P_{2}\left(z-z^{\prime}\right)
$$

In the case of a single-frequency $(\omega)$ mode, the $P$-representation of an oscillator of the same frequency in the coherent state $|\zeta\rangle$ is clearly given from Eq. (18) as

$$
P_{1}(z)=\pi \delta^{(2)}(z-\zeta)
$$

Furthermore, Glauber has shown ${ }^{2}$ that the $P$-representation of the corresponding mode of a random incoherent field, with average number of photons $\bar{n}$, is given by

$$
P_{2}(z)=(1 / \bar{n}) \exp \left(-|z|^{2} / \bar{n}\right) \text {. }
$$

Equations (43), (44), and (45) hence yield that the $P$-representation for the admixture of an incoherent and coherent field is

$$
P(z)=(1 / \bar{n}) \exp \left(-|z-\zeta|^{2} / \bar{n}\right),
$$

which provides the basis for the Glauber-Lachs formalism ${ }^{2,3}$ in quantum optics.

In our case we have essentially, for a single-frequency $(\omega)$ mode, a superposition of a coherent field in a state $|\zeta\rangle$ with a thermal (incoherent) black-body radiation field at temperature $T$. The mean number of thermal photons, $n_{T}$, in the thermal field itself,

$$
n_{T}=\left\langle\hat{n}_{0}\right\rangle_{0} \equiv \operatorname{Tr}\left(\hat{\rho}_{0}(\beta) \hat{n}_{0}\right)=\left(\mathrm{e}^{\beta \hbar \omega}-1\right)^{-1},
$$

which is simply the usual Planck distribution. Equations (44)-(47) then show the expected agreement with our previously proven $P$-representation for $\hat{\rho}(z ; \beta)$ given by Eq. (39). We can similarly show rather easily that the mean number of bosons in our mixed coherent state $\hat{\rho}(\zeta ; \beta)$ is given by the sum of the number $n_{T}$ from the incoherent thermal field and the number $|\zeta|^{2}$ from the pure coherent source,

$$
n_{T}(z)=\left\langle\hat{n}_{0}\right\rangle_{\zeta} \equiv \operatorname{Tr}\left(\hat{\rho}(\zeta ; \beta) \hat{n}_{0}\right)=n_{T}+|\zeta|^{2} .
$$




\subsection{Generalized $P$ - and Q-representations for nonzero temperatures}

As we have already seen in Sec. 1, the formalism of $P$ - and $Q$-representations is based on the (pure) Glauber coherent states. Although the latter form an overcomplete basis they are of practical usefulness due to the existence of a resolution of the identity operator in terms of them, as given by Eq. (13). Thus, since our new mixed coherent states $\hat{\rho}(z ; \beta)$ generalize the pure coherent states to nonzero temperatures, $T \neq 0$, and also provide a resolution of the identity operator given by Eq. (41), we are naturally led to enquire whether we can generalize the $P$ - and $Q$-representations of an arbitrary operator $\hat{\Theta}$ to the $T \neq 0$ case, in terms of them.

In the first place an obvious generalization to the $T \neq 0$ case of the $(T=0)$ $Q$-representation of Eq. (17) is

$$
Q(\hat{\Theta} ; z ; \beta) \equiv \operatorname{Tr}\{\hat{\rho}(z ; \beta) \hat{\Theta}\},
$$

and as the corresponding generalization of the $P$-representation we hypothesize that an arbitrary trace-class operator permits the expansion

$$
\hat{\Theta} \equiv \iint_{C} \frac{\mathrm{d}^{2} z}{\pi} P(\hat{\Theta} ; z ; \beta) \hat{\rho}(z ; \beta)
$$

It is clear that both $Q(\hat{\Theta} ; z ; \beta)$ and $P(\hat{\Theta} ; z ; \beta)$ reduce to their previous purestate counterparts $Q(\hat{\Theta} ; z)$ and $P(\hat{\Theta} ; z)$ respectively in the zero-temperature limit, $\beta \rightarrow \infty$. Equations (49) and (50) yield immediately the following relation between the generalized $P$ - and $Q$-representations of an arbitrary operator $\hat{\Theta}$,

$$
Q(\hat{\Theta} ; z ; \beta)=\iint_{C} \frac{\mathrm{d}^{2} z^{\prime}}{\pi} \operatorname{Tr}\left\{\hat{\rho}(z ; \beta) \hat{\rho}\left(z^{\prime} ; \beta^{\prime}\right)\right\} P\left(\hat{\Theta} ; z^{\prime} ; \beta^{\prime}\right)
$$

By making use of the general relation

$$
\operatorname{Tr}\left\{\hat{\Theta}_{1} \hat{\Theta}_{2}\right\}=\iint_{C} \frac{\mathrm{d}^{2} z}{\pi} P\left(\hat{\Theta}_{1} ; z\right) Q\left(\hat{\Theta}_{2} ; z\right),
$$

which follows immediately from Eqs. (17) and (18), we may use Eqs. (38) and (39) to derive the result,

$$
\begin{aligned}
\operatorname{Tr}\left\{\hat{\rho}(z ; \beta) \hat{\rho}\left(z^{\prime} ; \beta^{\prime}\right)\right\} & =\frac{2}{g\left(\beta, \beta^{\prime}\right)} \exp \left\{-\frac{2\left|z-z^{\prime}\right|^{2}}{g\left(\beta, \beta^{\prime}\right)}\right\}, \\
g\left(\beta, \beta^{\prime}\right) & \equiv \frac{\sinh \left[\frac{1}{2}\left(\beta+\beta^{\prime}\right) \hbar \omega\right]}{\sinh \left(\frac{1}{2} \beta \hbar \omega\right) \sinh \left(\frac{1}{2} \beta^{\prime} \hbar \omega\right)} .
\end{aligned}
$$

Equations (51) and (53) then yield, as the $T \neq 0$ generalization of Eq. (29),

$$
\begin{aligned}
Q(\hat{\Theta} ; z ; \beta) & =\exp \left[+\frac{1}{8} g\left(\beta, \beta^{\prime}\right) \Delta_{z}\right] P\left(\hat{\Theta} ; z ; \beta^{\prime}\right) \Longleftrightarrow \\
\tilde{Q}(\hat{\Theta} ; w ; \beta) & =\exp \left[-\frac{1}{8} g\left(\beta, \beta^{\prime}\right)|w|^{2}\right] \tilde{P}\left(\hat{\Theta} ; w ; \beta^{\prime}\right) .
\end{aligned}
$$

By taking the limit $\beta^{\prime} \rightarrow \infty$ in Eq. (55), and by making use of Eq. (29), we find

$$
\tilde{Q}(\hat{\Theta} ; w ; \beta)=\exp \left[-\frac{1}{4}\left(\mathrm{e}^{\beta \hbar \omega}-1\right)^{-1}|w|^{2}\right] \tilde{Q}(\hat{\Theta} ; w) \text {. }
$$


By making repeated use of Eqs. (55) and (56) we may also prove the relations

$$
\begin{aligned}
& \tilde{Q}(\hat{\Theta} ; w ; \beta)=\exp \left[-\frac{1}{8} g\left(\beta,-\beta^{\prime}\right)|w|^{2}\right] \tilde{Q}\left(\hat{\Theta} ; w ; \beta^{\prime}\right) \\
& \tilde{P}(\hat{\Theta} ; w ; \beta)=\exp \left[+\frac{1}{8} g\left(\beta,-\beta^{\prime}\right)|w|^{2}\right] \tilde{P}\left(\hat{\Theta} ; w ; \beta^{\prime}\right)
\end{aligned}
$$

Finally, by using the above relations together with Eq. (28), we also find the very important relations

$$
\begin{aligned}
& \tilde{Q}(\hat{\Theta} ; w ; \beta)=\exp \left[-\frac{1}{8} \operatorname{coth}\left(\frac{1}{2} \beta \hbar \omega\right)|w|^{2}\right] \tilde{W}(\hat{\Theta} ; w) \\
& \tilde{P}(\hat{\Theta} ; w ; \beta)=\exp \left[+\frac{1}{8} \operatorname{coth}\left(\frac{1}{2} \beta \hbar \omega\right)|w|^{2}\right] \tilde{W}(\hat{\Theta} ; w)
\end{aligned}
$$

Equations (59) and (60) show the formal result that the generalized $(T \neq 0)$ $P$-representation may be viewed as the analytic continuation to negative temperatures of the corresponding generalized $Q$-representation, and vice versa. We find this unexpected result both fascinating and suggestive. It surely merits further investigation.

\section{4. (Squeezed) SU(1,1) Pure Coherent States}

Having introduced our thermal mixed coherent states in Sec. 3, it is natural to consider next whether it might be possible to generalize them to a broader class. To this end we first discuss a separate class of pure coherent states built on the $\mathrm{SU}(1,1)$ group rather than the Heisenberg-Weyl group that underpins the Glauber coherent states. The SU $(1,1)$ Lie algebra is based on the three operators $\left\{\hat{K}_{x}, \hat{K}_{y}, \hat{K}_{z}\right\}$ obeying the commutation relations,

$$
\left[\hat{K}_{x}, \hat{K}_{y}\right]=-\mathrm{i} \hat{K}_{z} ;\left[\hat{K}_{y}, \hat{K}_{z}\right]=+\mathbf{i} \hat{K}_{x} ;\left[\hat{K}_{z}, \hat{K}_{x}\right]=+\mathbf{i} \hat{K}_{y}
$$

or, equivalently, on the three operators $\left\{\hat{K}_{ \pm} \equiv \hat{K}_{x} \pm \mathrm{i} \hat{K}_{y}, \hat{K}_{0} \equiv \hat{K}_{z}\right\}$ obeying

$$
\left[\hat{K}_{0}, \hat{K}_{ \pm}\right]= \pm \hat{K}_{ \pm} ;\left[\hat{K}_{+}, \hat{K}_{-}\right]=-2 \hat{K}_{0}
$$

The $\mathrm{SU}(1,1)$ Casimir operator is given by

$$
\hat{K}^{2} \equiv \hat{K}_{z}^{2}-\hat{K}_{x}^{2}-\hat{K}_{y}^{2}=\hat{K}_{0}^{2}-\frac{1}{2}\left(\hat{K}_{+} \hat{K}_{-}+\hat{K}_{-} \hat{K}_{+}\right) .
$$

The standard $\mathrm{SU}(1,1)$ basis (for $k>\frac{1}{2}$ is then $\{|k ; \mu\rangle ; \mu=k+n ; n=0,1,2, \ldots\}$ with

$$
\hat{K}^{2}|k ; \mu\rangle=k(k-1)|k ; \mu\rangle ; \hat{K}_{0}|k ; \mu\rangle=\mu|k ; \mu\rangle
$$

The group $\mathrm{SU}(1,1)$ is non-compact, and consequently there is no further restriction in general on the value of $k \in \mathbb{R}$. As a consequence the group has several series of irreducible unitary representations. In particular, there exist two so-called discrete series of representations, $T^{+}$and $T^{-}$, of which it is sufficient to consider only one, say $T^{+}$, since all the results clearly carry over to the other case under the substitution $k \rightarrow 1-k$. The representations of $T^{+}$are characterized by the values $k=\frac{1}{2}, 1, \frac{3}{2}, \ldots$. 
The SU(1,1) group generators now involve the so-called squeezing operators. By making use of various disentangling relations for the group they may be written in a number of equivalent ways as follows,

$$
\begin{aligned}
\hat{S}(z) \equiv \hat{S}(t, \phi) & \equiv \exp \left(\mathrm{i} \phi \hat{K}_{z}\right) \exp \left(\mathrm{i} t \hat{K}_{y}\right) \exp \left(-\mathrm{i} \phi \hat{K}_{z}\right) \\
& =\exp \left[\frac{1}{2} t\left(\mathrm{e}^{\mathrm{i} \phi} \hat{K}_{+}-\mathrm{e}^{-\mathrm{i} \phi} \hat{K}_{-}\right)\right] \equiv \exp (\mathrm{i} t \mathbf{n} \cdot \hat{\mathbf{K}}) \\
& =\exp \left(z \hat{K}_{+}\right) \exp \left[\ln \left(1-|z|^{2}\right) \hat{K}_{0}\right] \exp \left(-z^{*} \hat{K}_{-}\right)
\end{aligned}
$$

where

$$
\begin{aligned}
& z \equiv \tanh \left(\frac{1}{2} t\right) \exp (\mathrm{i} \phi) ; t, \phi \in \mathbb{R}, 0 \leq t<\infty, 0 \leq \phi<2 \pi \\
& \mathbf{n} \equiv(\sin \phi, \cos \phi, 0)
\end{aligned}
$$

So far our discussion has been completely abstract and general. However, for further progress we must consider specific realizations of the $\mathrm{SU}(1,1)$ algebra. In the remainder of this Section we consider three such realizations, each involving harmonic oscillators.

\subsection{Squeezed states of the one-mode oscillator}

A common realization of the $\mathrm{SU}(1,1)$ algebra arises in the context of single-mode squeezed-pair states, where it is easy to check that the three operators

$$
\hat{K}_{+} \rightarrow \frac{1}{2}\left(\hat{a}_{0}^{\dagger}\right)^{2} ; \hat{K}_{-} \rightarrow \frac{1}{2}\left(\hat{a}_{0}\right)^{2} ; \hat{K}_{0} \rightarrow \frac{1}{2}\left(\hat{a}_{0}^{\dagger} \hat{a}_{0}+\frac{1}{2}\right),
$$

obey the commutation relations of Eq. (62), with the Casimir operator of Eq. (63) now given by $\hat{K}^{2}=-\frac{3}{16} \hat{1}$, such that this becomes the $k=\left(\frac{1}{4}, \frac{3}{4}\right)$ representation of $\mathrm{SU}(1,1)$. The mode of action of the group operators of Eq. (65) on the underlying boson operators is the expected homogeneous Bogoliubov transformation to squeezed bosons,

$$
\hat{S}(z) \hat{a}_{0} \hat{S}^{\dagger}(z)=\left(1-|z|^{2}\right)^{-1 / 2}\left(\hat{a}_{0}-z \hat{a}_{0}^{\dagger}\right) \equiv \hat{b}_{z} .
$$

\section{2. $k$-squeezed states of the one-mode oscillator}

The $\mathrm{SU}(1,1)$ algebra can also be realized in the context of the above single-mode oscillator in a different way,

$$
\hat{K}_{+} \rightarrow \hat{a}_{0}^{\dagger}\left(\hat{n}_{0}+2 k \hat{1}\right)^{1 / 2} ; \hat{K}_{-} \rightarrow\left(\hat{n}_{0}+2 k \hat{\mathbb{1}}\right)^{1 / 2} \hat{a}_{0} ; \hat{K}_{0} \rightarrow \hat{n}_{0}+k \hat{1}
$$

which is based on the SU(1,1) analogue of Holstein-Primakoff theory ${ }^{4}$ for spin systems obeying the SU(2) algebra. These operators give a general $k$-realization of $\mathrm{SU}(1,1)$, as is readily verified from explicit evaluation of their commutation relations. If we let the group squeezing operators of Eq. (65) in this $k$-realization of Eq. (69), $\hat{S}_{k}(z)$, act on the oscillator (or bosonic) vacuum state we generate a new 
class of coherent states, now associated with the group $\mathrm{SU}(1,1)$,

$$
\begin{aligned}
|z ; k\rangle & \equiv \hat{S}_{k}(z)|0\rangle \\
& =\left(1-|z|^{2}\right)^{k} \sum_{n=0}^{\infty}[p(k, n)]^{1 / 2} z^{n}|n\rangle ;|z|<1,
\end{aligned}
$$

where

$$
p(k, n) \equiv \frac{\Gamma(n+2 k)}{\Gamma(n+1) \Gamma(2 k)}
$$

which were first introduced by Perelomov ${ }^{5}$ in a general context, and later studied by one of us $^{6}$ in the present harmonic oscillator context. They are defined in the unit disk $D \equiv\{z ;|z|<1\}$, and they play a similar role for the $\mathrm{SU}(1,1)$ group as do the Glauber coherent states for the Heisenberg-Weyl group. The boson-number distribution of the states $|z ; k\rangle$ is the negative-binomial distribution,

$$
p_{n}\left(|z|^{2} ; k\right) \equiv|\langle n \mid z ; k\rangle|^{2}=\left(1-|z|^{2}\right)^{2 k}|z|^{2 n} p(k, n),
$$

and for this reason the states $|z ; k\rangle$ have been called ${ }^{6}$ negative-binomial states. We note that for the special case $k=\frac{1}{2}$ we get the thermal (Planck) distribution of Eqs. $(35,36)$,

$$
p_{n}\left(|z|^{2} ; \frac{1}{2}\right)=p_{n}(\beta) ;|z|^{2}=\mathrm{e}^{-\beta \hbar \omega} .
$$

The states $\left|z ; \frac{1}{2}\right\rangle$ are the so-called harmonic oscillator phase (or angle) states. ${ }^{6}$ One can also simply show that in the limit that $k \rightarrow \infty, z \rightarrow 0, \sqrt{2 k} z=\zeta=$ const., the negative-binomial coherent state $|z ; k\rangle$ becomes equal to the Glauber coherent state $|\zeta\rangle$.

Very importantly for our purposes, these pure negative-binomial states are also coherent states, because they provide an easily proven resolution of the identity operator,

$$
\frac{(2 k-1)}{\pi} \iint_{D} \mathrm{~d} \mu(z)|z ; k\rangle\langle z ; k|=\hat{\mathbb{1}} ; k>\frac{1}{2}
$$

in terms of the invariant measure on $D$,

$$
\mathrm{d} \mu(z)=\left(1-|z|^{2}\right)^{-2} \mathrm{~d}^{2} z,
$$

which is just the expected Lobachewsky metric for the hyperbolic geometry of $\mathrm{SU}(1,1)$. The case $k=\frac{1}{2}$ is handled as the limiting case $k=\frac{1}{2}+\epsilon$, in the limit as $\epsilon \rightarrow 0^{+}$. The analogue of Eq. (16), which holds for the Glauber coherent states, for the overlap of two negative-binomial coherent states is

$$
\left\langle z ; k \mid z^{\prime} ; k\right\rangle=\left(1-|z|^{2}\right)^{k}\left(1-\left|z^{\prime}\right|^{2}\right)^{k}\left(1-z^{*} z^{\prime}\right)^{-2 k}
$$




\subsection{Squeezed states of the two-mode oscillator}

We now consider a two-mode harmonic oscillator Hilbert space $H_{1} \otimes H_{2}$, in which $\hat{a}_{1}^{\dagger} \in H_{1}$ and $\hat{a}_{2}^{\dagger} \in H_{2}$ are the creation operators of the two modes (or bosons). We also consider the joint number eigenstates, $|m, n\rangle \equiv|m\rangle_{1}|n\rangle_{2}$, and the following subspaces $H_{[k]}$ and $H_{[k]}^{\prime}$ spanned by them,

$$
\begin{aligned}
H_{[k]} & \equiv\{|n+2 k-1, n\rangle ; k \text { fixed }, n=0,1,2, \ldots\}, \\
H_{[k]}^{\prime} & \equiv\{|n, n+2 k-1\rangle ; k \text { fixed, } n=0,1,2, \ldots\}, \\
k & =\frac{1}{2}, 1, \frac{3}{2}, \ldots, \\
H_{1} \otimes H_{2} & =H_{[1 / 2]} \oplus H_{[1]} \oplus H_{[1]}^{\prime} \oplus H_{[3 / 2]} \oplus H_{[3 / 2]}^{\prime} \oplus \cdots .
\end{aligned}
$$

We may now readily check that the three operators

$$
\hat{K}_{+} \rightarrow \hat{a}_{1}^{\dagger} \hat{a}_{2}^{\dagger} ; \hat{K}_{-} \rightarrow \hat{a}_{1} \hat{a}_{2} ; \hat{K}_{0} \rightarrow \frac{1}{2}\left(\hat{a}_{1}^{\dagger} \hat{a}_{1}+\hat{a}_{2}^{\dagger} \hat{a}_{2}+\frac{1}{2}\right),
$$

obey the commutation relations of Eq. (62), with the Casimir operator of Eq. (63) now given by

$$
\hat{K}^{2} \rightarrow \frac{1}{4}\left(\hat{a}_{1}^{\dagger} \hat{a}_{1}-\hat{a}_{2}^{\dagger} \hat{a}_{2}\right)^{2}-\frac{1}{4} .
$$

In the above Hilbert subspaces $H_{[k]}$ and $H_{[k]}^{\prime}$ with $k=\frac{1}{2}, 1, \frac{3}{2}, \ldots$, the standard $\mathrm{SU}(1,1)$ basis vectors of Eq. (64) are respectively given by

$$
|k ; \mu \equiv k+n\rangle \rightarrow|n+2 k-1, n\rangle \in H_{[k]},|n, n+2 k-1\rangle \in H_{[k]}^{\prime} .
$$

It is easily seen that the corresponding two-mode squeezing operators of Eq. (65) in this realization of the $\mathrm{SU}(1,1)$ algebra commute with the projection operators onto the subspaces $H_{[k]}$ and $H_{[k]}^{\prime}$, and thus leave these Hilbert subspaces invariant.

The mode of action of the group operators of Eq. (65) on the underlying two boson creation operators is now the Bogoliubov transformation to squeezed twomode bosons,

$$
\begin{aligned}
& \hat{S}(z) \hat{a}_{1} \hat{S}^{\dagger}(z)=\left(1-|z|^{2}\right)^{-1 / 2}\left(\hat{a}_{1}-z \hat{a}_{2}^{\dagger}\right) \equiv \hat{b}_{z}^{(1)}, \\
& \hat{S}(z) \hat{a}_{2} \hat{S}^{\dagger}(z)=\left(1-|z|^{2}\right)^{-1 / 2}\left(\hat{a}_{2}-z \hat{a}_{1}^{\dagger}\right) \equiv \hat{b}_{z}^{(2)} .
\end{aligned}
$$

Finally, if we let the squeezing operator in this realization act on either the lowest state $|2 k-1,0\rangle \in H_{[k]}$ or the analogous state $|0,2 k-1\rangle \in H_{[k]}^{\prime}$, we generate the corresponding two-mode SU $(1,1)$ coherent states. In the former case, for example,

$$
\begin{aligned}
|z ; l, 0\rangle & \equiv \hat{S}(z)|l, 0\rangle ; \quad l=0,1,2, \ldots \\
& =\left(1-|z|^{2}\right)^{(1+l) / 2} \sum_{n=0}^{\infty}\left[\frac{(n+l) !}{n ! l !}\right]^{1 / 2} z^{n}|n+l, n\rangle ; \quad|z|<1,
\end{aligned}
$$

which should be compared with Eqs. $(70,71)$ for the corresponding one-mode $k$ squeezed state, when $l=2 k-1$. These states clearly obey the same completeness relation of Eqs. $(74,75)$ with $\left|z ; \frac{1}{2}(1+l)\right\rangle \rightarrow|z ; l, 0\rangle$. 


\section{Displaced Negative-Binomial Mixed Coherent States}

We now return to our second realization of the $\mathrm{SU}(1,1)$ algebra from Sec. 4.2 above and the associated two-boson $k$-squeezed states of the one-mode oscillator. Since these provide a generalization of our earlier Glauber coherent states, we now attempt $^{7}$ a similar generalization to a new class of mixed coherent states which, if they exist, will generalize our earlier thermal mixed coherent states. To that end we first introduce the density operator for the negative-binomial mixed states,

$$
\hat{\rho}(k, r) \equiv(1-r)^{2 k} \sum_{n=0}^{\infty} p(k, n) r^{n}|n\rangle\langle n| ; \quad 0 \leq r \leq 1 ; k=\frac{1}{2}, 1, \frac{3}{2}, \ldots,
$$

where $p(k, n)$ is as given previously in Eq. (71). The special case $k=\frac{1}{2}$ of Eq. (83) simply reduces to our earlier thermal oscillator mixed state of Eq. (32),

$$
\begin{aligned}
\hat{\rho}_{\mathrm{th}}(r) \equiv \hat{\rho}\left(k=\frac{1}{2}, r\right) & =(1-r) \sum_{n=0}^{\infty} r^{n}|n\rangle\langle n| \\
& =\left(1-\mathrm{e}^{-\beta \hbar \omega}\right) \exp \left(-\beta \hbar \omega \hat{a}_{0}^{\dagger} \hat{a}_{0}\right) ; \beta \hbar \omega=-\ln r .
\end{aligned}
$$

The average number of bosons in the negative-binomial mixed state is given by

$$
n(k, r)=\left\langle\hat{n}_{0}\right\rangle_{k, r} \equiv \operatorname{Tr}\left(\hat{\rho}(k, r) \hat{n}_{0}\right)=2 k\left(\frac{1}{r}-1\right)^{-1},
$$

which reduces to the thermal Planck distribution of Eq. (47) in the special case $k=\frac{1}{2}$.

We note that $\hat{\rho}(k, r)$ may also be written in terms of the thermal density operator,

$$
\hat{\rho}(k, r)=[(2 k-1) !]^{-1}\left(\frac{1}{r}-1\right)^{2 k-1}\left(\hat{a}_{0}\right)^{2 k-1} \hat{\rho}_{\mathrm{th}}(r)\left(\hat{a}_{0}^{\dagger}\right)^{2 k-1} .
$$

It is also of interest to note that one can easily show from Eq. (70) that the negativebinomial mixed state $\hat{\rho}(k, r)$ can be obtained from the corresponding pure-state projector by randomizing the phase of the argument $z$,

$$
\hat{\rho}(k, r)=\int_{0}^{2 \pi} \frac{\mathrm{d} \theta}{2 \pi}|z ; k\rangle\langle z ; k| ; z \equiv \sqrt{r} \exp \mathrm{i} \theta
$$

Finally, by analogy with our procedure for generating the mixed thermal coherent states in Sec. 3, we now displace $\hat{\rho}(k, r)$, using the Weyl displacement operator of Eq. (9), to yield the displaced negative-binomial mixed (coherent) states,

$$
\begin{aligned}
\hat{\rho}(z ; k, r) & \equiv \hat{D}(z) \hat{\rho}(k, r) \hat{D}^{\dagger}(z) \\
& =(1-r)^{2 k} \sum_{n=0}^{\infty} p(k, n) r^{n}|n ; z\rangle\langle n ; z| ; 0 \leq r \leq 1,
\end{aligned}
$$

which reduces to Eq. (35), as expected in the special case $k=\frac{1}{2}$. In the special case $r=0, \mathrm{Eq}$. (88) reduces to the pure Glauber coherent state,

$$
\hat{\rho}(z ; k=0, r)=|z\rangle\langle z| \text {. }
$$


We can also show rather easily that the mean number of bosons in our displaced negative-binomial mixed coherent state $\hat{\rho}(\zeta ; k, r)$ is given by

$$
n(\zeta ; k, r)=\left\langle\hat{n}_{0}\right\rangle_{\zeta ; k, r} \equiv \operatorname{Tr}\left(\hat{\rho}(\zeta ; k, r) \hat{n}_{0}\right)=2 k\left(\frac{1}{r}-1\right)^{-1}+|\zeta|^{2},
$$

which may be compared with its thermal counterpart of Eq. (48). Just as in Eq. (41) for the mixed thermal coherent states, so we can prove ${ }^{7}$ the analogous very important relation that the negative-binomial mixed states also provide a resolution of the identity operator,

$$
\iint_{C} \frac{\mathrm{d}^{2} z}{\pi} \hat{\rho}(z ; k, r)=\hat{\mathbf{l}}
$$

which shows that they may also be regarded as mixed coherent states, and thus provide the desired generalization of their thermal counterparts. There are many ways in which Eq. (91) may be proved. Perhaps the most direct way is to take matrix elements in the number-state basis, and use the definition of Eq. (88) together with the well-known and easily proven form of the matrix elements of the displacement operator in terms of the associated Laguerre polynomials $L_{n}^{l-n}(x) .^{7}$

In view of the existence of the completeness relation of Eq. (91) it is clear that everything we did in Sec. 3 for the thermal coherent states $\left(k=\frac{1}{2}\right)$ can now be generalized to the present case $k=\frac{1}{2}, 1, \frac{3}{2}, \ldots$ For example, we can give further $(k, r)$-extensions of the ordinary $P$ - and $Q$-representations as the obvious generalizations of Eqs. $(49,50)$,

$$
\begin{aligned}
Q(\hat{\Theta} ; z ; k, r) & \equiv \operatorname{Tr}(\hat{\rho}(z ; k, r) \hat{\Theta}) \\
\hat{\Theta} & \equiv \iint_{C} \frac{\mathrm{d}^{2} z}{\pi} P(\hat{\Theta} ; z ; k, r) \hat{\rho}(z ; k, r)
\end{aligned}
$$

Clearly the case $r=0$ of Eq. (92) just reproduces the ordinary $P$ - and $Q$ representations of Eqs. $(17,18)$, while the case $\left\{k=\frac{1}{2}, \beta \hbar \omega=-\ln r\right\}$ gives our previous thermal results of Eqs. $(49,50)$. Typical of the many interesting relations that we find are the following,

$$
\tilde{Q}(\hat{\Theta} ; w ; k, r)=\exp \left[-\frac{|w|^{2}}{8} \frac{(1+r)}{(1-r)}\right] L_{2 k-1}\left(\frac{|w|^{2} r}{4(1-r)}\right) \tilde{W}(\hat{\Theta} ; w)
$$

where $L_{n}(x)$ is a Laguerre polynomial, and

$$
\tilde{P}(\hat{\Theta} ; w ; k, r)=\exp \left[+\frac{|w|^{2}}{8} \frac{(1+r)}{(1-r)}\right]\left[L_{2 k-1}\left(\frac{|w|^{2} r}{4(1-r)}\right)\right]^{-1} \tilde{W}(\hat{\Theta} ; w),
$$

which generalize Eqs. $(59,60)$, and

$$
\begin{aligned}
\tilde{Q}\left(\hat{\Theta} ; w ; k, r_{1}\right)= & \exp \left[-\frac{|w|^{2}}{4} \frac{\left(1-r_{1} r_{2}\right)}{\left(1-r_{1}\right)\left(1-r_{2}\right)}\right] L_{2 k-1}\left(\frac{|w|^{2} r_{1}}{4\left(1-r_{1}\right)}\right) \\
& \times L_{2 k-1}\left(\frac{|w|^{2} r_{2}}{4\left(1-r_{2}\right)}\right) \tilde{P}\left(\hat{\Theta} ; w ; k, r_{2}\right)
\end{aligned}
$$


which is a generalization of the pure (Glauber) coherent-state result of Eq. (29). A number of other similar results are discussed in more detail in Ref. [7].

Finally, we also note the existence of another resolution of the identity operator over the variable $r$, for fixed values of $z$ and $k$,

$$
(2 k-1) \int_{0}^{1} \mathrm{~d} r(1-r)^{-2} \hat{\rho}(z ; k, r)=\hat{\mathbb{1}} ; k>\frac{1}{2},
$$

where the case $k=\frac{1}{2}$ is again handled as the limiting case $k=\frac{1}{2}+\epsilon$, in the limit as $\epsilon \rightarrow 0^{+}$. Eq. (96) may be proven by making use of Eqs. (87) and (88) together with Eqs. (74) and (75). Although Eq. (96) seems not to have been exploited in the present context up till now, we believe that it should be profitable to give it further consideration.

\section{Generalized Thermo-Field Dynamics}

We turn finally to another application of the thermal and negative-binomial mixed states that we have discussed here. We recall the third realization of $\mathrm{SU}(1,1)$ that we introduced in Sec. 4.3 and the corresponding SU(1,1) two-mode pure coherent state of Eq. (82), with respective pure-state density operator in the two-boson Hilbert space $H_{1} \otimes H_{2}$ given by

$$
\hat{\rho}(l, 0 ; z) \equiv|z ; l, 0\rangle\langle z ; l, 0|; l=0,1,2, \ldots ;| z|<1 .
$$

If we take the partial trace of this density operator with respect to the first mode we find,

$$
\begin{aligned}
\hat{\rho}_{2}(l, 0 ; z) & \equiv \operatorname{Tr}_{1} \hat{\rho}(l, 0 ; z) \\
& =\hat{\rho}\left(k=\frac{1}{2}(1+l), r=|z|^{2}\right),
\end{aligned}
$$

which is simply our previous negative-binomial mixed state of Eq. (83). Similarly, by taking the partial trace with respect to the second mode we find,

$$
\begin{aligned}
\hat{\rho}_{1}(l, 0 ; z) & \equiv \operatorname{Tr}_{2} \hat{\rho}(l, 0 ; z) \\
& =\left(1-|z|^{2}\right)^{1+l} \sum_{n=0}^{\infty} \frac{(n+l) !}{n ! l !}|z|^{2 n}|n+l\rangle\langle n+l|
\end{aligned}
$$

which is simply a "shifted" negative-binomial mixed state, where the term "shifted" refers here to the fact that the series in Eq. (99) begins with the term proportional to $|l\rangle\langle l|$ rather than with the term proportional to $|0\rangle\langle 0|$. (Note also that this shifted negative-binomial mixed state should not be confused with the quite different displaced negative-binomial mixed state of Eq. (88.) Only in the special case $l=0$, i.e., $k=\frac{1}{2}$, do both partial traces agree with each other and yield the thermal density operator,

$$
\hat{\rho}_{1}(0,0 ; z)=\hat{\rho}_{2}(0,0 ; z)=\hat{\rho}\left(k=\frac{1}{2}, r=|z|^{2}\right) \equiv \hat{\rho}_{\mathrm{th}}\left(r=|z|^{2}\right) ;|z|^{2} \equiv \mathrm{e}^{-\beta \hbar \omega} .
$$


We now make use of the above formalism by letting $\hat{\Theta}_{2}$ be any operator acting in the Hilbert space $H_{2}$ and $\hat{\mathbb{1}}_{1}$ be the unit operator acting in $H_{1}$. Then we find,

$$
\begin{aligned}
\left\langle z ; l, 0\left|\hat{\mathbb{1}}_{1} \otimes \hat{\Theta}_{2}\right| z ; l, 0\right\rangle & =\operatorname{Tr}\left\{\left(\hat{\mathbb{1}}_{1} \otimes \hat{\Theta}_{2}\right) \hat{\rho}(l, 0 ; z)\right\}=\operatorname{Tr}_{2}\left\{\hat{\Theta}_{2} \hat{\rho}_{2}(l, 0 ; z)\right\} \\
& =\operatorname{Tr}\left\{\hat{\Theta} \hat{\rho}\left(k=\frac{1}{2}(1+l), r=|z|^{2}\right)\right\} .
\end{aligned}
$$

The special case $k=\frac{1}{2}$, (i.e., $l=0$ ) of Eq. (101) is the basic relation of thermo-field dynamics, ${ }^{8}$

$$
\left\langle z ; 0,0\left|\hat{\mathbb{1}}_{1} \otimes \hat{\Theta}_{2}\right| z ; 0,0\right\rangle=\operatorname{Tr}\left\{\hat{\Theta} \hat{\rho}_{\mathrm{th}}\left(|z|^{2}\right)\right\} ;|z|^{2}=\mathrm{e}^{-\beta \hbar \omega} .
$$

In thermo-field dynamics ${ }^{8}$ the first mode is the so-called "fictitious mode," and the two-mode $\mathrm{SU}(1,1)$ pure coherent state $|z ; 0,0\rangle \in H_{[1 / 2]}$ is the so-called "thermo-field vacuum." Essentially all of the results of thermo-field dynamics now follow from this last relation and the two-mode squeezing formalism presented here.

What has now been achieved by us in this context is to generalize the basic equation (102) of thermo-field dynamics to the much more general equation (101) of what we now call "negative-binomial-field dynamics." Whereas thermo-field dynamics is a formalism within the two-boson Hilbert subspace $H_{[1 / 2]}$, negative-binomial-field dynamics exists within the more general two-boson subspace $H_{[k]}$, where $k=\frac{1}{2}(1+l)$, associated with the $k$-representation of $\mathrm{SU}(1,1)$. We discuss possible uses of this formalism below.

\section{Discussion}

We have shown elsewhere ${ }^{9}$ how the thermal $(T \neq 0)$ coherent states of Sec. 3 may very profitably be used in the Bargmann representation, thereby extending the usual $(T=0)$ formalism. Clearly, the mixed thermal coherent-state formalism should be applicable in any situation where coherent signals co-exist with thermal noise. Examples abound in fields as diverse as quantum optics and quantum information theory. Other potential applications include signal and image processing, where the usual representations of a signal in the time domain or in the frequency domain are often less convenient than one in the joint time-frequency "phase space." Indeed there are many papers in the signal processing context that use the Wigner function method and allied techniques inspired by the Glauber coherent-state formalism. Wavelet approaches are just one such by-product.

We have also shown previously ${ }^{10}$ how the pure two-mode $\mathrm{SU}(1,1)$ coherent states of Sec. 4.3 arise in the context of a damped linear harmonic oscillator, and how they can be used for general such processes involving fluctuation-dissipation phenomena. The extension to the mixed case and negative-binomial-field dynamics will be interesting. In many practical applications the usefulness of our result of Eq. (101) will arise from the fact that the right-hand sides will often be easier to calculate than the corresponding left-hand sides. Moreover, in many physical situations the so-called "fictitious" mode will actually be quite real. A particular example is the so-called "accelerated observer formalism" investigated by us previously, ${ }^{11}$ where the two 
modes correspond to observers moving in opposite directions, each of whom only has a partial knowledge of the other due to being able to observe only a restricted region of space-time. This formalism provides a physically appealing account of the Hawking radiation from a black hole. There exist many other examples where we only have partial knowledge about the system, and where the "real" and "fictitious" modes represent simply the known and unknown parameters respectively. The unknown parameters are typically traced over, and what was originally a pure state in the "big" Hilbert space $H_{1} \otimes H_{2}$ becomes a mixed state in the "small" space $H_{1}$. In all such cases there will be a need for a basis of mixed states in the "small" space. Furthermore, such admixtures of coherence (pure states) with general noise (thermal or more general, such as negative-binomial) or decoherence is often essentially unavoidable in practice, and in all such situations the formalism presented here is potentially useful.

\section{References}

1. R.F. Bishop and A. Vourdas, J. Phys. A: Math. Gen. 20, 3743 (1987).

2. R.J. Glauber, Phys. Rev. 131, 2766 (1963).

3. G. Lachs, Phys. Rev. 138, B1012 (1965).

4. T. Holstein and H. Primakoff, Phys. Rev. 58, 1098 (1940).

5. A.M. Perelomov, Commun. Math. Phys. 26, 222 (1972).

6. A. Vourdas, Phys. Rev. A 45, 1943 (1992).

7. A. Vourdas and R.F. Bishop, Phys. Rev. A 51, 2353 (1995).

8. H. Umezawa, H. Matsumoto, and M. Tachiki, Thermo-Field Dynamics and Condensed States, (North-Holland, Amsterdam, 1987); S.M. Barnett and P.L. Knight, J. Opt. Soc. Am. B 2, 467 (1985).

9. A. Vourdas and R.F. Bishop, Phys. Rev. A 50, 3331 (1994).

10. R.F. Bishop and A. Vourdas, J. Phys. A: Math. Gen. 20, 3727 (1987).

11. R.F. Bishop and A. Vourdas, J. Phys. A: Math. Gen. 19, 2525 (1986). 